\title{
Value of chest radiography in phenotyping chronic obstructive pulmonary disease
}

\author{
M. Miniati*,\#, S. Monti ${ }^{\#}$, J. Stolk ${ }^{\star}$, G. Mirarchi ${ }^{\#}$, F. Falaschi', R. Rabinovich ${ }^{\S}$, \\ C. Canapini" ${ }^{\#}$, J. Roca ${ }^{\S}$ and K.F. Rabe
}

ABSTRACT: The objectives of the present study were to reappraise chest radiography for the diagnosis of emphysema, using computed tomography (CT) as the reference standard, and to establish whether or not chest radiography is useful for phenotyping chronic obstructive pulmonary disease (COPD).

Patients $(n=154)$ who had undergone posteroanterior and lateral chest radiography and CT for diagnostic purposes were studied. CT data were scored for emphysema using the picture-grading method. Chest radiographs were examined independently by five raters using four criteria for emphysema that had been validated against lung pathology. These criteria were then used to assess the prevalence of emphysema in 458 COPD patients. Patients with and without evidence of emphysema were compared with regard to age, sex, smoking history, body mass index (BMI), forced expiratory volume in one second (FEV1), diffusing capacity of the lung for carbon monoxide $(D L, C O)$ and health status.

Chest radiography yielded a sensitivity of $90 \%$ and a specificity of $98 \%$ for emphysema. Of the 458 COPD patients, 245 showed radiological evidence of emphysema. Emphysemic patients had a significantly lower BMI, FEV 1 and $D$ L,CO, greater restriction of physical activity and worse quality of life than nonemphysemic patients. There was no difference across the two groups with regard to age, sex or smoking history.

Chest radiography is a simple means of diagnosing moderate-to-severe emphysema. It is useful in phenotyping chronic obstructive pulmonary disease and may aid physicians in their choice of treatment.

KEYWORDS: Chest radiography, chronic obstructive pulmonary disease, computed tomography, emphysema

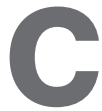
hronic obstructive pulmonary disease (COPD) is a major cause of morbidity, mortality and disability in the Western world [1].

Chronic airflow limitation is thought to result from the combination of two mechanisms: increased airway resistance due to narrowing of the small airways, and loss of lung elastic recoil due to emphysema. In clinical practice, spirometry plays a key role in the diagnosis of COPD and assessment of disease severity and progression [2]. However, it provides no information as to the presence or absence of emphysema. Since emphysema is defined as a structural pulmonary abnormality [3], its recognition is based on tests that reflect lung structure rather than function.
Computed tomography (CT) is currently the most accurate imaging technique for the diagnosis of emphysema in vivo [4]. Extensive use of this technique, however, seems unwarranted due to the high cost and substantial radiation burden. Conversely, chest radiography is invariably performed in patients with COPD, but its accuracy in diagnosing emphysema is controversial [5].

The aim of the present study was two-fold: 1) to reappraise chest radiography for the diagnosis or exclusion of emphysema using $\mathrm{CT}$ as the reference diagnostic standard; and 2) to establish whether or not chest radiography, as a simple means of detecting emphysema, may prove useful in phenotyping COPD.

Earn CME accreditation by answering questions about this article. You will find these at the back of the printed copy of this issue or online at http://www.erj.ersjournals.com/current.shtml

\section{AFFILIATIONS}

*Dept of Critical Care, University of Florence, Florence,

\#National Research Council Institute of Clinical Physiology, and

${ }^{+}$Diagnostic Radiology II, University Hospital, Pisa, Italy.

"Dept of Pulmonology, Leiden University Medical Centre, Leiden, the Netherlands.

${ }^{\S}$ Respiratory Service, Hospital Clinic Barcelona, Spain

CORRESPONDENCE

M. Miniati

Istituto di Fisiologia Clinica del CNR Via G. Moruzzi 1 56124 Pisa

Italy

Fax: 39503152166

E-mail: miniati@ifc.cnr.it

Received:

July 272007

Accepted after revision:

November 162007

\section{SUPPORT STATEMENT}

This study was supported, in part, by the European Union (Brussels, Belgium) Fifth Framework

Programme under contract number QLG1-CT-2001-01012 (COPD GENE SCAN Project). The funding source played no role in: study design; collection, analysis or interpretation of data; writing the report; or the decision to submit this article for publication.

STATEMENT OF INTEREST None declared. 


\section{MATERIAL AND METHODS \\ Chest radiography versus CT for the diagnosis of emphysema}

Sample

The study sample comprised 154 patients, who were evaluated at the Institute of Clinical Physiology (Pisa, Italy) between January 1, 2003 and December 31, 2004. In these patients, thoracic CT and posteroanterior and lateral chest radiography were performed for diagnostic purposes within 1 week of each other. The patients (124 males and 30 females) had a median (interquartile range) age of 62 (56-69) yrs. Of these, $135(88 \%)$ were either current or former smokers, with a smoking history of 44 (30-57) pack-yrs, and 107 (69\%) met the criteria of chronic airflow obstruction (forced expiratory volume in one second (FEV1)/forced vital capacity (FVC) of $<70 \%$ ). Fifty patients were evaluated for suspected lung cancer, 19 for suspected bronchiectasis, 11 for asbestos-related pleural thickening and one for suspected bronchiolitis obliterans. Of the remaining 73 patients, 48 were screened as candidates for lung volume reduction surgery for emphysema, 20 for liver transplantation and five for lung transplantation due to severe precapillary pulmonary hypertension.

\section{Computed tomography}

CT was performed using a Siemens Volume Zoom scanner (Siemens, Erlangen, Germany; 120 kV peak; 200 mA; scan time $1 \mathrm{~s})$, without infusion of contrast medium. The lungs were scanned from the apex through the base at 1-cm intervals with the patient in the supine position and breath-holding at full inspiration, using 1-mm collimation and a high-frequency spatial reconstruction algorithm. Scans were viewed using a window level of $-600 \mathrm{HU}$ and width of 1,500 HU, and were independently examined by two chest radiologists for the presence of areas of low attenuation and vascular disruption. The severity of emphysema was scored on a nonparametric scale from 0 (no emphysema) to 100 using the picture-grading method of THURLBECK et al. [6] adapted for CT [7]. According to this method, a score of $\leqslant 5$ is consistent with trace emphysema, a score of 10-30 indicates mild emphysema, a score of $>30-50$ moderate emphysema and a score of $>50$ severe emphysema.
Neither radiologist was aware of the patients' clinical data. The mean of two independent ratings was used for comparison with chest radiographic results.

\section{Chest radiography}

Posteroanterior and lateral chest radiography (99 digital and 55 analogue) were performed at a standard 2-m focus-to-detector (film) distance, with the patients upright and holding their breath at full inspiration. Chest radiographs were evaluated for the presence or absence of emphysema by five independent raters who had no access to clinical or CT data. Two of them were pulmonologists with experience in the interpretation of chest radiographs, and three (two medical students and one radiology technician) had limited or no experience. In evaluating emphysema, four radiographic criteria, originally introduced by SUTINEN et al. [8], were used. The radiographic criteria are described in table 1. According to SUTINEN et al. [8], a diagnosis of emphysema should be made when the chest radiographs reveal any two or more or these criteria. In their study, emphysema was correctly identified in ante-mortem chest radiographs in $82 \%$ of the patients who had autopsyproven emphysema. This represented any amount of emphysematous tissue destruction occupying $>2 \%$ of total lung volume as measured by the point-counting technique [10]. By contrast, all of the chest radiographs corresponding to the patients with structurally normal lungs were rated as normal. The overall accuracy of chest radiography for the diagnosis or exclusion of emphysema was $90 \%$.

The three less experienced raters were trained to identify the radiographic criteria of table 1 using a set of 25 chest radiographs from subjects with normal lungs and patients with emphysema of varying degree of severity. The training set did not include chest radiographs that were used for comparison with CT.

\section{Value of chest radiography in phenotyping COPD Sample}

The study sample included 458 patients with COPD who were part of a larger cohort enrolled in a case-control study that was

TABLE 1 Criteria for the radiographic diagnosis of emphysema

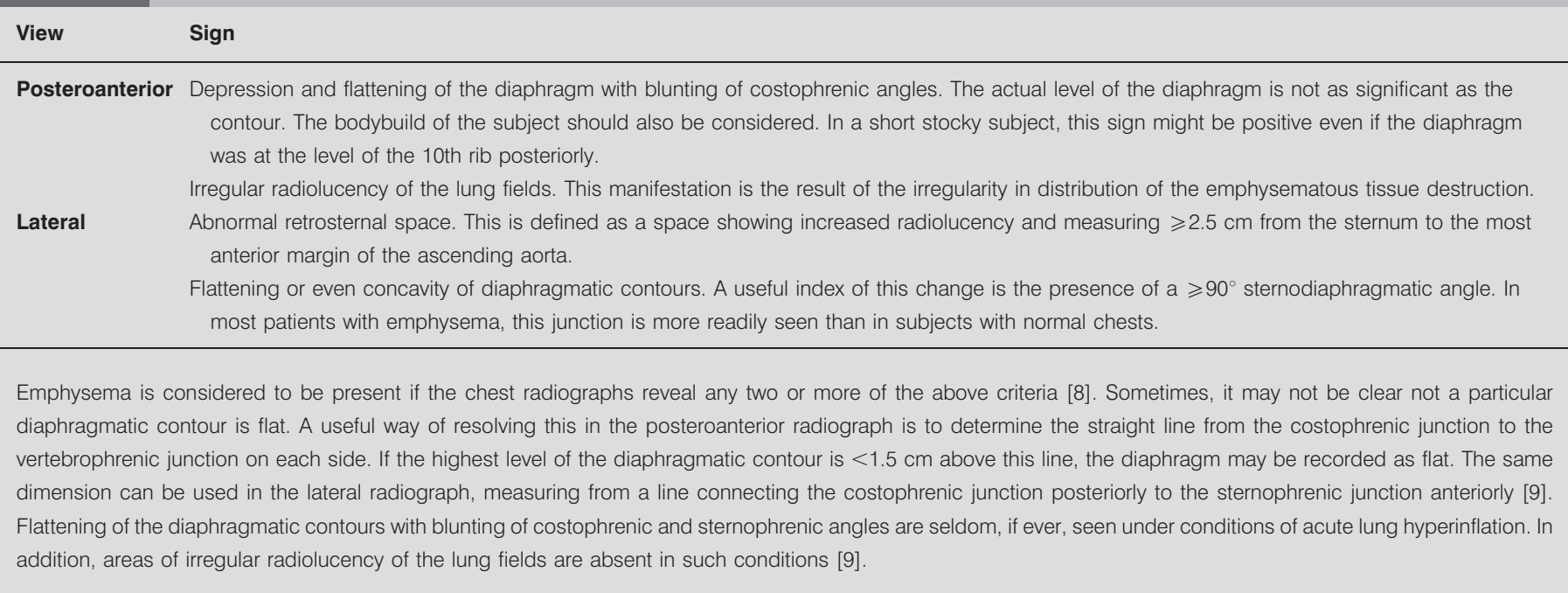


aimed at assessing genetic susceptibility for the development of COPD [11]. In that study, 1,018 COPD patients and 911 nondiseased controls were recruited at six European centres between October 1, 2001 and September 30, 2003. The 458 patients reported herein are those in whom posteroanterior and lateral chest radiography had been performed within a month before entering the study; 200 were recruited at the Institute of Clinical Physiology, 132 at the Respiratory Service, Hospital Clinic (Barcelona, Spain) and 126 at the Dept of Pulmonology, Leiden University Medical Centre (Leiden, the Netherlands).

\section{Patient selection criteria}

The criteria for patient recruitment were: a firm clinical diagnosis of stable COPD; airflow limitation as indicated by an $\mathrm{FEV} 1 / \mathrm{FVC}$ of $<70 \%$ and $\mathrm{FEV} 1$ of $\leqslant 70 \%$ of the predicted value [12]; reversibility of FEV1 on bronchodilation of $<12 \%$ or $<200 \mathrm{~mL}$; and a smoking history of $\geqslant 20$ pack-yrs.

Patients were excluded from the study if they had: an established diagnosis of asthma; established obstructive syndromes other than COPD; lung cancer; a history of atopy; known $\alpha_{1}$-antitrypsin deficiency; or a serum $\alpha_{1}$-antitrypsin concentration of $<1.0 \mathrm{~g} \cdot \mathrm{dL}^{-1}$. Patients were also excluded if they had experienced an acute exacerbation in the 4 weeks preceding study entry.

\section{Study protocol}

Clinical history-taking and physical examination were performed in all patients. Lung function studies included measurement of FVC and FEV1 (before and after bronchodilator), and single-breath diffusing capacity of the lung for carbon monoxide ( $D \mathrm{~L}, \mathrm{CO})$. Measured $D \mathrm{~L}, \mathrm{CO}$ were corrected for haemoglobin and carbon monoxide levels and expressed as a percentage of the predicted value [13]. Patients were then invited to complete a self-administered questionnaire (St George's Respiratory Questionnaire), which consists of three sections: symptoms, activity and impacts [14]. The symptoms section is concerned with the frequency of cough, mucus hypersecretion, wheeze and breathlessness. The activity section deals with the physical activities that either cause or are limited by breathlessness. The impacts section covers such factors as disturbance of daily life, expectations for health, employment, and need for medication and its side-effects. Each of the three sections is scored separately in the range 0$100 \%$. The total score, which utilises the responses to all items, also ranges $0-100 \%$.

The protocol was approved by the local ethics committees. Before entering the study, informed written consent was obtained from all patients.

\section{Chest radiography}

The chest radiographs (272 digital and 186 analogue) were examined independently by the two pulmonologists who were involved in the first part of the study. Emphysema was diagnosed if any two or more of the criteria in table 1 were present. In a subset of 200 cases, the chest radiographs were also used to measure total lung capacity (TLC) according to the planimetric method of HARRIS et al. [15]. Software was developed that provided online computation of TLC from digital chest radiographs. Radiological TLC was expressed as $\%$ pred using appropriate reference equations [16].

\section{Statistical analysis}

Inter-rater reliability in scoring emphysema on CT was tested for by means of intraclass correlation [17]. Inter-rater agreement in diagnosing or excluding emphysema on chest radiography was assessed by means of the kappa statistic [18]. The standard normal distribution was used for testing whether inter-observer agreement was better than chance [18], and 95\% confidence intervals (CIs) were calculated according to the binomial distribution. The relation between severity of airflow obstruction or number of chest radiographic criteria for emphysema and CT emphysema score was assessed using the Kruskal-Wallis nonparametric test. Patients' characteristics across the two diagnostic groups (emphysema versus no emphysema) were compared using contingency tables for categorical variables (Fisher's exact test). For the continuous variables, differences between the two groups were tested for
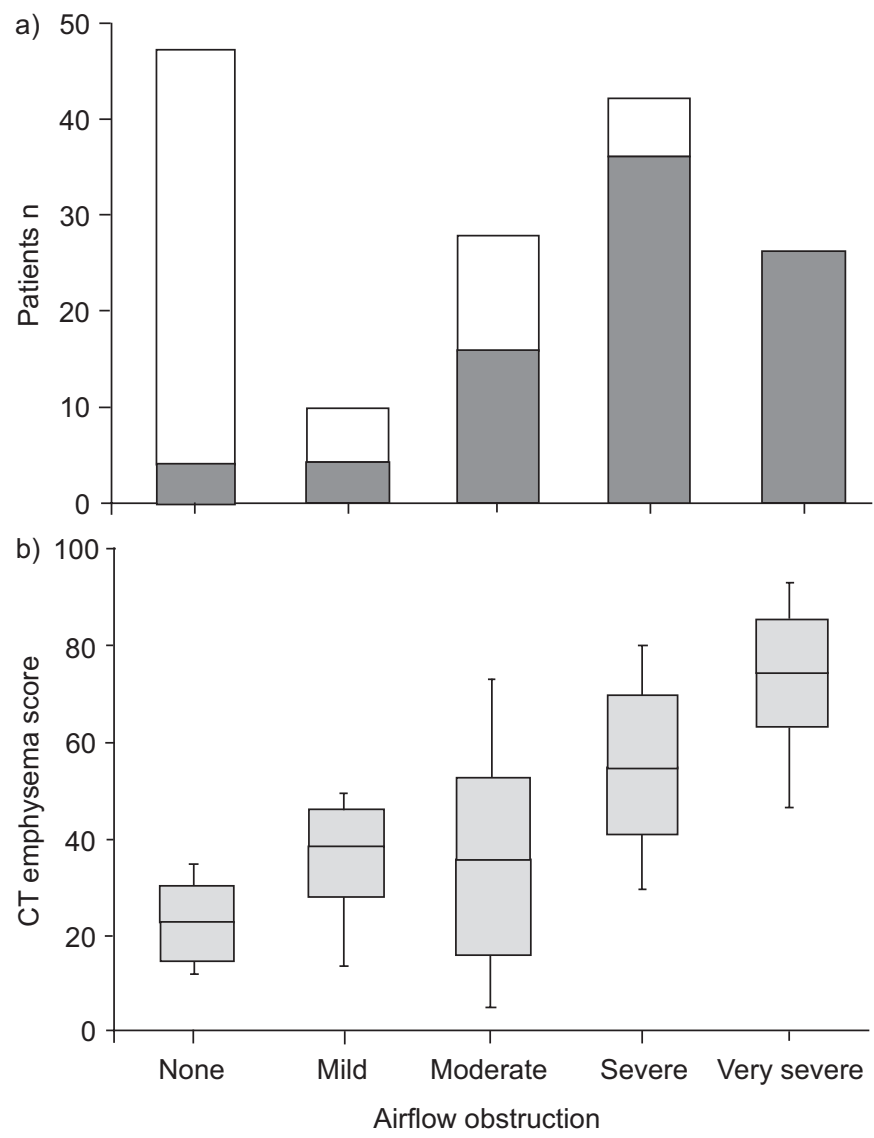

FIGURE 1. a) Prevalence of emphysema ( $\square$ : absent; $\square$ : present) on computed tomography $(\mathrm{CT})$ as a function of airflow obstruction (mild: forced expiratory volume in one second (FEV 1 )/forced vital capacity $<70 \%$ and $F E V_{1}$ $\geqslant 80 \%$ of the predicted value; moderate: FEV $1 \geqslant 50-<80 \%$ pred; severe: FEV 1 $\geqslant 30-<50 \%$ pred; very severe: $\mathrm{FEV}_{1}<30 \%$ pred) in 154 patients. b) Boxplot showing CT emphysema score (mean of two independent ratings) as a function of airflow obstruction in 87 patients with CT-confirmed emphysema. Boxes represent median (50th percentile) and interquartile range; vertical bars represent the 10th and 90th percentiles. $p<0.0001$ (Kruskal-Wallis nonparametric test) 


\begin{tabular}{|c|c|c|c|c|}
\hline TABI & $\begin{array}{l}\text { Chest ra } \\
\text { tomogra } \\
\text { emphys }\end{array}$ & $\begin{array}{l}\text { ography ve } \\
\text { רy (CT) for } \\
\text { רa }\end{array}$ & $\begin{array}{l}\text { iagnosis or ex } \\
\text { iagnosited }\end{array}$ & usion of \\
\hline \multirow{2}{*}{$\begin{array}{l}\text { Rater } \\
\text { no. }\end{array}$} & \multicolumn{2}{|c|}{ Emphysema on $\mathrm{CT}^{\#}$} & \multicolumn{2}{|c|}{ No emphysema on $\mathrm{CT}^{\circ}$} \\
\hline & $\begin{array}{c}\text { True positive } \\
\mathbf{n}\end{array}$ & $\begin{array}{c}\text { Sensitivity } \\
\%\end{array}$ & $\begin{array}{c}\text { True negative } \\
\mathbf{n}\end{array}$ & $\begin{array}{c}\text { Specificity } \\
\%\end{array}$ \\
\hline 1 & 79 & 91 & 66 & 99 \\
\hline 2 & 78 & 90 & 66 & 99 \\
\hline 3 & 78 & 90 & 66 & 99 \\
\hline 4 & 78 & 90 & 65 & 97 \\
\hline 5 & 79 & 91 & 65 & 97 \\
\hline
\end{tabular}

Raters 1 and 2 were pulmonologists, raters 3 and 4 were medical students and rater 5 was a radiology technician. Two patients were rated false positive for emphysema based on depression and flattening of diaphragmatic contours in posteroanterior and lateral projections. One was an 81-yr-old male with severe airflow obstruction (forced expiratory volume in one second (FEV 1 ) of $46 \%$ predicted); CT revealed extensive vascular disruption in lower lobes without clear areas of low attenuation. The other was an 86-yr-old male with no airflow obstruction (FEV1 of $103 \%$ pred); CT did not show areas of low attenuation. ${ }^{\#}: \mathrm{n}=87 ;{ }^{\bullet}: \mathrm{n}=67$.

using the Mann-Whitney nonparametric test. Two-tailed pvalues of $<0.05$ were considered significant throughout.

\section{RESULTS}

\section{Chest radiography versus $\mathrm{CT}$ in the diagnosis of emphysema}

Emphysema was diagnosed consistently by two independent radiologists in $87(56 \%)$ of $154 \mathrm{CT}$ scans. The median CT emphysema score was 60 (interquartile range 38-74). Intraclass correlation yielded a coefficient of 0.9987 (95\% CI $0.9983-$ 0.9991), indicating excellent inter-rater reliability in scoring emphysema on CT. The prevalence of emphysema in the sample increased as a function of the severity of airflow obstruction (fig. 1a). Similarly, CT emphysema scores were significantly higher in patients with severe or very severe airflow obstruction than in those with mild or moderate obstruction (fig. 1b).

Adopting the radiographic criteria of table 1, the five independent raters agreed that emphysema was present in 77 patients and absent in 70. Overall inter-rater agreement was 95\% (147 out of 154) and kappa 0.95 (95\% CI 0.90-1.00), indicating that inter-rater agreement was significantly better than chance $(p<0.0001)$.

The sensitivity and specificity of chest radiography for the diagnosis or exclusion of emphysema are reported in table 2 for each of the five independent raters. The weighted sensitivity was $90 \%$ (95\% CI $87-93 \%)$ and the weighted specificity 98 (96-99)\%. The positive predictive value was 98 (96-99)\% and the negative predictive value 88 (85-91)\%.

In the 87 patients with CT-confirmed emphysema, there was a highly significant positive relation between the number of chest radiographic criteria for emphysema and CT score (fig. 2).

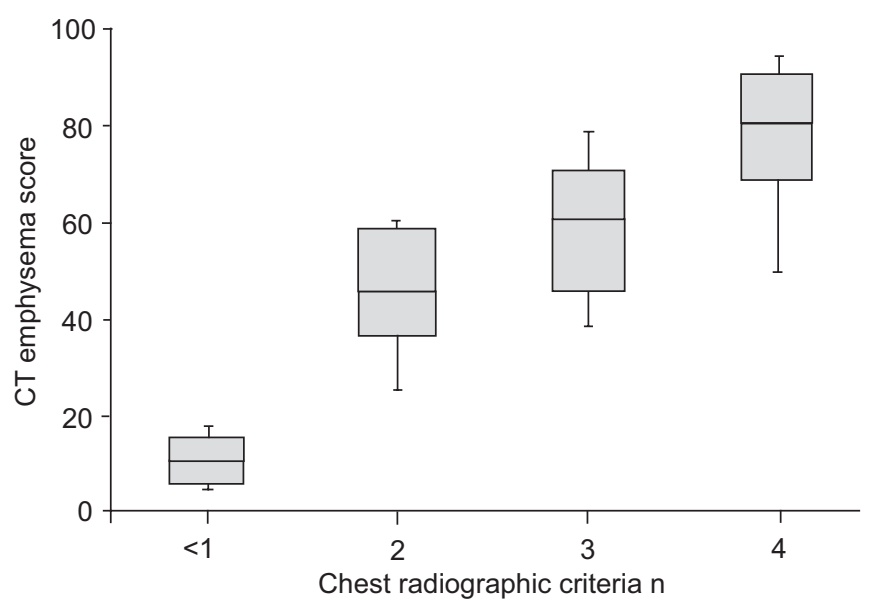

FIGURE 2. Boxplot showing computed tomography (CT) emphysema score (mean of two independent ratings) as a function of the number of chest radiographic criteria for emphysema (median of five independent ratings) in 87 patients with CTconfirmed emphysema. Boxes represent median (50th percentile) and interquartile range; vertical bars represent the 10th and 90th percentiles $(\leqslant 1: n=10 ; 2: n=20 ; 3$ : $n=28 ; 4: n=29) . p<0.0001$ (Kruskal-Wallis nonparametric test).

Table 3 reports the CT scores, type of emphysema and regional distribution of emphysematous lesions in the 11 patients whose chest radiographs had been rated as negative for emphysema by at least one of the five independent observers. The CT scores were consistent with trace or mild emphysema.

\section{Value of chest radiography in phenotyping COPD}

The baseline characteristics of the 458 patients with COPD are given in table 4 .

Emphysema was diagnosed consistently by the two independent raters in $245(53 \%)$ out of 458 patients. Patients with emphysema did not differ from those without emphysema with regard to age, sex or, surprisingly, smoking history. By contrast, body mass index (BMI), FEV1 and DL,CO were significantly lower in patients with emphysema than in those without. Among the 200 patients in whom radiological TLC was measured, those whose chest radiographs met the criteria for emphysema had a significantly higher TLC than those without emphysema.

Regarding the results of the St Georges Respiratory Questionnaire, there was no difference between the two groups in terms of frequency of respiratory symptoms. However, emphysemic patients showed significantly greater limitation of their physical activity and worse quality of life than nonemphysemic patients.

\section{DISCUSSION}

The value of chest radiography for the assessment of emphysema has been a matter of contention since the 1960s [10]. In radiological-pathological correlation studies, the agreement between chest radiograph interpretation and morphological findings ranges from excellent [8] to poor [19], depending upon the radiographic criteria used and the strictness applied by the investigators in matching their interpretation to the presence or absence of structural emphysema [10]. 


\begin{tabular}{lccc}
$\begin{array}{l}\text { TABLE } \mathbf{3} \\
\text { Patient } \\
\text { no. }\end{array}$ & $\begin{array}{c}\text { False-negative ratings on chest radiography } \\
\text { negative }\end{array}$ & $\begin{array}{c}\text { CT emphysema } \\
\text { score }\end{array}$ & $\begin{array}{c}\text { Type of } \\
\text { emphysema }\end{array}$ \\
\hline $\mathbf{1}$ & 5 & 5 & Paraseptal \\
$\mathbf{2}$ & 5 & 5 & Paraseptal \\
$\mathbf{3}$ & 4 & 5 & Paraseptal \\
$\mathbf{4}$ & 5 & 5 & Paraseptal \\
$\mathbf{5}$ & 3 & 10 & Paraseptal \\
$\mathbf{6}$ & 5 & 10 & Centrilobular \\
$\mathbf{7}$ & 3 & 15 & Centrilobular \\
$\mathbf{8}$ & 5 & 15 & Centrilobular \\
$\mathbf{9}$ & 3 & 15 & Centrilobular \\
$\mathbf{1 0}$ & 3 & 20 & Centrilobular \\
$\mathbf{1 1}$ & 1 & 25 & Centrilobular \\
\hline
\end{tabular}

All patients were assessed by each of the five raters. All emphysema was located in the upper lobe. CT: computed tomography.

Even though CT is now regarded as the most accurate imaging technique for detecting emphysema, it is questionable whether it should be performed for the specific purpose of diagnosing emphysema due to the high cost and substantial radiation exposure. Standard-dose multidetector CT of the thorax yields an effective radiation dose of 6-8 $\mathrm{mSv}$ [20]. By contrast, digital chest radiography entails a much lower radiation burden than CT (0.04-0.07 mSv for posteroanterior and lateral chest radiography), is far less expensive and ubiquitously available.

Therefore, the present study was undertaken in order to reappraise chest radiography as a simple means of diagnosing or excluding emphysema. In doing so, four radiographic criteria that had been validated against lung pathology [8] were used. Since the study from which these criteria are derived was published long before the introduction of $\mathrm{CT}$, it was thought reasonable to test the validity of such criteria against this newer imaging modality.

Emphysema was diagnosed on chest radiographs in most patients with CT-confirmed disease (sensitivity 90\%). However, chest radiography failed to detect trace or mild emphysema that was apparent on CT. The rate of false-positive results was very low (specificity 98\%). These findings are remarkable inasmuch as three out of five independent raters had very limited experience in interpreting chest radiographs.

The high inter-rater agreement may be explained as follows. First, the radiological diagnosis of emphysema is based primarily upon the evaluation of the shape of the lungs rather than on signs of vascular attenuation that are barely recognised by inexperienced clinicians or technologists. Secondly, the diagnosis of emphysema requires that at least two of the four radiological criteria be present, and this helps to reduce interobserver variability. Thirdly, the less experienced raters were trained to recognise chest radiographic abnormalities using an appropriate set of standards. Fourthly, all the patients were studied under stable clinical conditions.

However, it is acknowledged that the inter-rater agreement reported in the present study may not be easily replicated.

\begin{tabular}{|c|c|c|c|c|}
\hline \multirow[t]{2}{*}{ TABLE 4} & \multicolumn{4}{|c|}{$\begin{array}{l}\text { Baseline characteristics of the chronic } \\
\text { obstructive pulmonary disease patients }\end{array}$} \\
\hline & Total & Emphysema & No emphysema & p-value ${ }^{\#}$ \\
\hline Subjects n & 458 & 245 & 213 & \\
\hline Male sex & 375 (82) & $204(83)$ & $171(80)$ & 0.48 \\
\hline Age yrs & $66(61-71)$ & $66(61-71)$ & $66(60-70)$ & 0.54 \\
\hline $\begin{array}{l}\text { Smoking } \\
\text { pack-yrs }\end{array}$ & $46(37-57)$ & $46(37-58)$ & $45(35-57)$ & 0.42 \\
\hline BMI $\mathbf{k g} \cdot \mathrm{m}^{-2}$ & 27 (24-30) & 25 (22-28) & 29 (26-32) & $<0.0001$ \\
\hline FEV $1 \%$ pred & $48(36-60)$ & $42(31-51)$ & 56 (44-65) & $<0.0001$ \\
\hline DL,CO \% pred & $66(50-80)$ & 55 (40-67) & 78 (64-92) & $<0.0001$ \\
\hline $\begin{array}{c}\text { TLCRad \% } \\
\text { pred }\end{array}$ & $114(104-122)$ & $122(112-134)$ & 107 (99-116) & $<0.0001$ \\
\hline \multicolumn{5}{|l|}{ SGRQ score \% } \\
\hline Symptoms & $37(20-55)$ & $40(21-59)$ & $35(20-53)$ & 0.16 \\
\hline Activity & 54 (42-79) & $59(48-80)$ & $53(36-67)$ & 0.0004 \\
\hline Impacts & $34(18-51)$ & 39 (22-53) & $29(13-46)$ & $<0.0001$ \\
\hline Total & $41(27-57)$ & $45(30-61)$ & $37(25-51)$ & $<0.0001$ \\
\hline
\end{tabular}

Data are presented as $\mathrm{n}(\%)$ or median (interquartile range), unless otherwise indicated. BMI: body mass index; FEV1: forced expiratory volume in one second; \% pred: \% predicted; DL,CO: diffusing capacity of the lung for carbon monoxide; TLCRad: radiological total lung capacity; SGRQ: St George's Respiratory Questionnaire. * : emphysema versus no emphysema; ": available for 200 patients (82 with emphysema and 118 without).

Therefore, it would be desirable that the validity of the proposed radiological criteria be tested in different clinical settings.

In the sample of 154 patients with a $56 \%$ prevalence of emphysema, chest radiography yielded a positive predictive value of $98 \%$ and a negative predictive value of $88 \%$. Epidemiological surveys in samples of the Italian general population indicate that the prevalence of chronic obstructive lung disease in subjects aged $\geqslant 50 \mathrm{yrs}$ is $\sim 30 \%$ [21]. It might thus be hypothesised that the prevalence of structural emphysema lies in the range $10-15 \%$. In this range, chest radiography would yield a positive predictive value of $\sim 86 \%$ and a negative predictive value of close to $99 \%$.

The present results differ from those of THURLBECK and SIMON [19], who examined the value of chest radiography by comparing radiograph interpretation with that of inflation-fixed paper-mounted lung specimens. The radiographs were interpreted by one rater only and the diagnosis of emphysema was made whenever characteristic vascular changes, termed arterial deficiency, were seen. This criterion permitted correct diagnosis in only $16 \%$ of the patients with mild-to-moderate emphysema and $42 \%$ of those with moderately severe-to-severe emphysema. The specificity of chest radiography was $98 \%$.

Strictly speaking, the results reported by THURLBECK and SIMON [19] apply to the single rater involved and to the use of a single radiological criterion, arterial deficiency. Therefore, they have no bearing on the results of the present study, in which different criteria were used. 
The second objective was to establish whether or not scoring chest radiographs for the presence or absence of emphysema might help in the characterisation of the COPD phenotype. Patients who met the radiological criteria for emphysema had a significantly lower BMI, FEV1 and DL,CO than those who did not. Conversely, radiological TLC was significantly higher in patients with emphysema than in those without. This lends support to the validity of the criteria used, which primarily reflect lung hyperinflation [8]. In addition, emphysemic patients experienced greater restriction of physical activity and had a much worse quality of life than nonemphysemic patients.

Optimal bronchodilation is recommended as the first step in the management of stable COPD [2]. However, substantial improvements in exercise tolerance, symptoms and health-related quality of life are often achieved only after the implementation of pulmonary rehabilitation [22]. Randomised trials are, therefore, needed in order to establish whether or not rehabilitation programmes are as effective or more effective than standard pharmacological treatment in emphysemic patients.

Correct recognition of emphysema on chest radiography would be equally valuable in patients who do not have or are not known to have COPD. This is quite likely to occur in clinical practice because chest radiographs are often taken for reasons other than a chronic respiratory illness. If the chest radiograph of one such patient meets the criteria for emphysema, it is likely that the disease is present and the patient should be tested for airflow obstruction. Should the patient be a smoker, the diagnosis of emphysema would be a particularly strong indication for giving up smoking since the lungs of such patients are overtly damaged by inhaled smoke.

In summary, the results of the present study indicate that chest radiography is a valuable, inexpensive means of diagnosing moderate-to-severe emphysema. However, it is less sensitive than computed tomography for the detection of mild emphysema and less accurate for the evaluation of the regional distribution of emphysema.

\section{ACKNOWLEDGEMENTS}

The authors wish to thank G. Catapano, L. Manfredi, E. Fornai and C. Carli for clinical and technical assistance.

\section{REFERENCES}

1 Mannino DM, Homa DM, Akinbami LJ, Moorman JE, Gwynn C, Redd SC. Chronic obstructive pulmonary disease surveillance - United States, 1971-2000. MMWR Surveill Summ 2002; 51: 1-16.

2 Celli BR, MacNee W, committee members. Standards for the diagnosis and treatment of patients with COPD: a summary of the ATS/ERS position paper. Eur Respir J 2004; 23: 932-943.

3 Anon, Ciba Guest Symposium Report. Terminology, definitions, and classification of chronic pulmonary emphysema and related conditions. Thorax 1959; 14: 286-299.
4 Coxson HO, Rogers RM. Quantitative computed tomography of chronic obstructive pulmonary disease. Acad Radiol 2005; 12: 1457-1463.

5 Thurlbeck WM. Emphysema then and now. Can Respir J 1994; 1: 21-39.

6 Thurlbeck WM, Dunnill MS, Hartung W, Heard BE, Heppleston AG, Ryder RC. A comparison of three methods of measuring emphysema. Hum Pathol 1970; 1: 215-226.

7 Kuwano K, Matsuba K, Ikeda T, et al. The diagnosis of mild emphysema. Correlation of computed tomography and pathology scores. Am Rev Respir Dis 1990; 141: 169-178.

8 Sutinen S, Christoforidis AJ, Klugh GA, Pratt PC. Roentgenologic criteria for the recognition of nonsymptomatic pulmonary emphysema. Correlation between roentgenologic findings and pulmonary pathology. Am Rev Respir Dis 1965; 91: 69-76.

9 Pratt PC. Role of conventional chest radiography in diagnosis and exclusion of emphysema. Am J Med 1987; 82: 998-1006.

10 Dunnill MS. Quantitative methods in the study of pulmonary pathology. Thorax 1962; 17: 320-328.

11 Chappell S, Daly L, Morgan K, et al. Cryptic haplotypes of SERPINA1 confer susceptibility to chronic obstructive pulmonary disease. Hum Mutat 2006; 27: 103-109.

12 Crapo RO, Morris AH, Gardner RM. Reference spirometric values using equipment and technique that meet ATS recommendations. Am Rev Respir Dis 1981; 123: 659-664.

13 Cotes JE, Chinn DJ, Quanier PH, Roca J, Yernault JC. Standardization of the measurement of transfer factor (diffusing capacity). Eur Respir J 1993; 6: Suppl. 16, 41-52.

14 Jones PW, Quirk FH, Baveystock CM, Littlejohn P. A selfcomplete measure of heath status for chronic airflow limitation. The St. George's Respiratory Questionnaire. Am Rev Respir Dis 1992; 145: 1321-1327.

15 Harris TR, Pratt PC, Kilburn KH. Total lung capacity measured by roentgenograms. Am J Med 1971; 50: 756-763.

16 O'Brien RJ, Drizd TA. Roentgenographic determination of total lung capacity: normal values from a national population survey. Am Rev Respir Dis 1983; 126: 949-952.

17 Shrout PE, Fleiss JL. Intraclass correlations: uses in assessing rater reliability. Psychol Bull 1979; 86: 420-427.

18 Fleiss JL. Measuring nominal scale agreement among many raters. Psychol Bull 1971; 76: 378-382.

19 Thurlbeck WM, Simon G. Radiographic appearance of the chest in emphysema. AJR Am J Roentgenol 1978; 130: 429-440.

20 Tack D, Gevenois PA. Radiation dose in computed tomography of the chest. In: BankierA, Genevois PA, eds. Imaging. Eur Respir Mon 2004; 9: 39-50.

21 Viegi G, Matteelli G, Angino A, et al. The proportional Venn diagram of obstructive lung disease in the Italian general population. Chest 2004; 126: 1093-1101.

22 Troosters T, Casaburi R, Gosselink R, Decramer M. Pulmonary rehabilitation in chronic obstructive pulmonary disease. Am J Respir Crit Care Med 2005; 172: 19-38. 